\title{
THE MACHINE VISION BLIND GUIDE SYSTEM
}

\author{
Yun-Long LaY ${ }^{1}$, Pei-Wen Chen ${ }^{2}$, HUI-JEN YANG ${ }^{3}$ \\ ${ }^{1}$ Department of Electronic Engineering, National Chin-Yi Institute of Technology, \\ ${ }^{2}$ Department of Electronic Engineering, Nan Kai College, \\ ${ }^{3}$ Department of Information Management, National Chin-Yi Institute of Technology, \\ Taichung, Taiwan
}

\begin{abstract}
The available guide tools of the orientation and mobility for a blind are the cane, guide dog and electronic guide devices. A cane is easy to detect the hindrance that is in front of the user but not for the hindrance above the user waist. That's why a cane user of a blind sometimes will be hit by the upper hindrance. Guide dog is a very powerful mobility guider but expensive and the training and living care for the dogs are difficult. Hence, guide dog is not popular in many countries. The electronic devices for blind guide tools such as laser cane; sonic glasses, sonic guide etc. can only detect a single point at a time and not for a whole view. In our system, a machine vision blind guide system is proposed. A CCD grabbed the image of front view and divided the image into nine blocks. Each block is calculated to get the distance message, which is multipoint data to guide the blind by the converted voice signal.
\end{abstract}

Biomed Eng Appl Basis Comm, 2002 (April): 14: 81-85.

Keywords: blind guide, machine vision, distance measurement

\section{INTRODUCTION}

A visual hindrance child cannot tell the relative position of the environment where he is staying. The lack acknowledgement of the space cause he feels difficult to move from one place to the other certain place. Hence, it is easy to understand how fear for an untrained visual handicap to do the orientation and mobility. If parents limit the mobility of the visual handicap kid for safety consideration, no doubt the kid will lose the learning chance of motion independent and increase the living dependency [1].

Received: Nov. 5, 2001; accepted: March 20, 2002

Correspondence: Yun-Long Lay, Professor

Department of Electronic Engineering

National Chin-Yi Institute of Technology

No.35, Lane 215, Sec. 1, Chung-Shan Rd., Taiping, Taichung, 411 Taiwan

Email: layyl@chinyi.ncit.edu.tw
The current assistant tools of orientation and mobility for visual handicap are cane, guide dog, and some electronic device [2]. The user age of cane is after 14. A blind uses cane to detect the hindrance before moving step and warms the un-blind people. The weak point of a cane is not convenient to detect the hindrance over waist. Most blind uses cane to detect lower hindrance in front of moving step. They are possible hurt by the higher hindrance.

The guide dog is a powerful guider for a blind. The user age of a guide dog is about 17 or older not suitable for elementary student, who is too young to control the dog. The guide dog is very expensive, the training and living care are not easy. The available electronic guide tools for a blind are laser cane, sonic glasses, and sonic guide etc. Those non-contact distance measurement tools are dominated by ultrasound and laser two types. These electronic measuring devices transmit narrow beams of sound or light waves that "bounce" off selected solid objects back to the hand-held receiver. Custom electronics and a microprocessor convert and calculate the elapsed time into a 
comprehendible distance measurement unit transmitted the voice message by an earphone $[3,4]$.

It's quite easy to assess the distance using one of the aforesaid distance meters, but each measurement only gets a single point distance data. In this proposed system, a multi-point machine vision blind guide has been developed. When the image in front of the user is grabbed by the system, nine areas divide the image. Each area is calculated by the computer for getting the distance data and transmits to the user's ear by a voice conversion with nine data each image frame. A blind using this system will roughly understand the distances of the hindrances, which is a whole frame not a single point, in front of the moving step.

\section{METHOD}

For a given optical system, when a point image on a film or CCD is blurring as a small enough size due to defocusing within the depth of focus but not adversely affects the performance of the system [5]. The depth of focus is the amount by which the image may be shifted longitudinally with respect to some reference plane. The depth of field is the amount by which the object may be shifted before the acceptable blur is produced. The size of the acceptable blur may be specified as the linear diameter of the blur spot or as an angular blur. Hence, an object is focused on the image plane where has a short range to move as a focus tolerance for a clear image. In other word, an image is clear when the focus mechanism is set within the depth of focus and the image is getting unclear by out of the depth of focus.

A simple experiment will show us that no matter the object complicated or not, the image still has the entire high and low frequency response. Fig. I has shown us that similar patters have the similar frequency response. Fig. 2 a,b,c are the frequency response of Fig.l a that is taken from the same pattern with different focus distance. Fig. $2 \mathrm{a}$ is a right focus image, Fig.2b is a little bit out of focus, and Fig. $2 \mathrm{c}$ is more out of focus. The difference of those three frequency responses is that the correct focus image has the high frequency of the $200 \mathrm{lp} / \mathrm{mm}$ but not the out of focus images. Fig. $1 \mathrm{~b}$ is a correct focus image of a very simple pattern with only a single bar but the frequency response has all the high frequency expansion as shown in Fig.2d. Our proposed method calculated the frequency response of the transfer function of an image. The transfer function is as a low pass filter when the image more out of focus the worse the high frequency response.

From Gauss object-image formula [6]

$$
\frac{1}{d_{n}}+\frac{1}{d}=\frac{1}{f}
$$

where

$d_{n}$ : object distance from principle plane of the lens to object.

$d_{1}$ : image distance from principle plane of the lens to image plane.

$f:$ focus distance.

For a clear image, the focus distance of the lens and the image distance are known then the object distance can be calculated easily. That's the algorithm we use for measuring the object distance. Base on the Fourier Optics theory [7-9], we can represent the object-image relation for a right focus system by the following formula,

$$
t_{0}=p_{0} * h_{0}
$$

where $*$ is the convolution operator

$t_{0}:$ right focus image

$p_{0}:$ object,

$h_{0}$ : system impulse response of the right focus image

For an out of focus image, the object-image relation would be as equation (3).

$$
t_{1}=p_{1} * h_{1}
$$

$t$, : out of focus image

$p_{1}$ : out of focus object, where $i=1,2,3 \ldots \ldots, i \rightarrow N$

The larger the $\mathrm{i}$ value, the more the image out of focus. We could represent $p_{1}$ as $p_{1}=p_{0} * s$, where $s_{1}$ is defocus coefficient.

An out of focus image could be written as:

$$
t_{1}=\left(p_{0} * s,\right) * h_{0}=p_{0} *\left(s, * h_{0}\right)=p_{0} * h,
$$

where $h_{1}$ : the system impulse response of an out of focus image,

$$
h_{1}=s_{1} * h_{0}
$$

From equation (2) to (4), it is easy to know that adjusting the focus ring of the lens is equal to change the amount of $h$, for the same object at the same distance. When the focus distance match equation (1), the image should be very clear. We analyzed the frequency response of those image in different focus distance and found that the high frequency response is better for the right focus image than the out of focus image.

The equation (2) to (4) would be derived as (5) and (6) after taking the Fourier transform.

$$
T_{0}=P_{0} \cdot H_{0}, H_{0}=\frac{T_{0}}{P_{0}}
$$




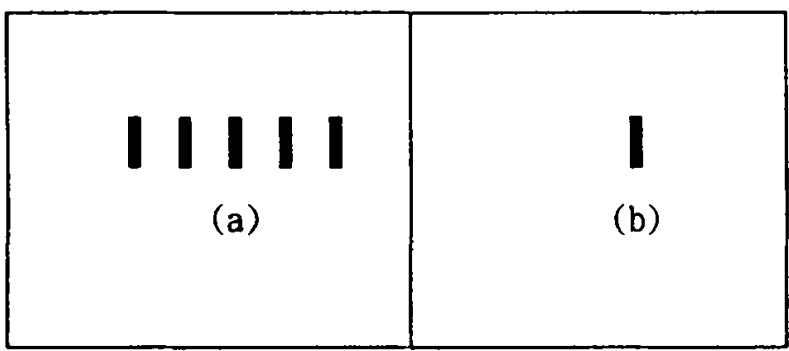

Fig. 1 A sample grating

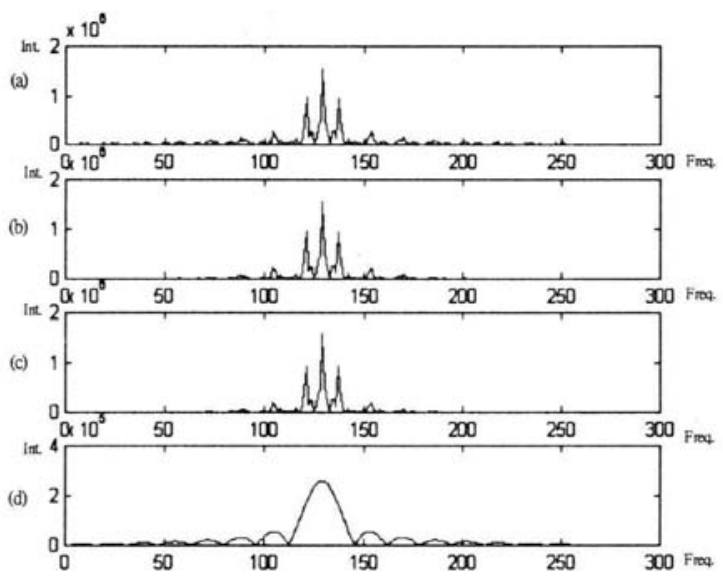

Fig.2 The frequency response of the gratings

$$
T_{1}=P_{0} \cdot H_{1}, H_{1}=\frac{T_{t}}{P_{0}}
$$

where $T_{1}, H_{i}$ is the form of $t_{1}, h_{1}$ after Fourier transform.

The frequency response of $H_{0}$ is wider than the frequency response of $H_{1}$. Comparing all the different $H_{1}$, it is easy to choose a cut off frequency as the frequency threshold for defining the right focus image. Hence, the $f_{c u t}$ of an image has the highest frequency response possessed the best clear image.

Fig. 3 is a testing image got in the lab where 9 IC components are put in front of a CCD camera with different distance between the camera lens and those components. The focus ring has set and let the middle component to be the right focus distance but not the rest components. The image is converted into 256 gray scales and finds the skeleton as the edges feature by edge processing algorithm. Divide the image into 9 medium-blocks, from top to bottom, left to right, the order is named from 1 to 9 shown as Fig.4. Each block of the 9 medium-blocks has been divided into 9 smallblocks and label the small-blocks from a to i. All the small-blocks should be processed by Fourier transform to calculate the highest frequency response as shown in Fig.5. For each medium-block, whose 9 small-

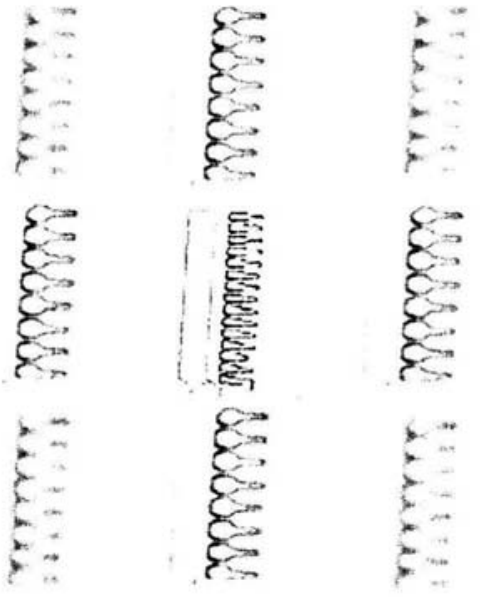

Fig.3 The image pattern of different distance objects

\begin{tabular}{|c|c|c|c|c|}
\hline $\mathrm{a}$ & $\mathrm{b}$ & $\mathrm{c}$ & & \\
\hline $\mathrm{d}$ & $\mathrm{e}$ & $\mathrm{f}$ & 2 & 3 \\
\hline $\mathrm{g}$ & $\mathrm{h}$ & $\mathrm{i}$ & & \\
\hline & & & & \\
\hline & 4 & 5 & 6 \\
\hline & & & & \\
\hline & & 8 & 9 \\
\hline
\end{tabular}

Fig.4 The division method of an image

blocks have the most blocks of high frequency response then the medium-block is the best focus image block. The highest frequency response block is the clearest image block, which is the middle mediumblock and named 5 as shown in Fig.6. In other word, the number 5 medium-block has the most small-blocks of high frequency response. Hence, the highest average intensity of each medium-block is the number 5 block shown as Fig.6. The vertical axis of Fig. 6 is the average intensity of the nine small blocks. The horizontal axis of Fig.6 is frequency response using the $1 \mathrm{p} / \mathrm{mm}$ unit.

\section{TESTING RESULTS}

In our system to measure a distance by a certain image, all the image slices from infinite (10 meter) to nearest should be recorded once $20 \mathrm{~cm}$ by adjusting the lens and analysis those images to check which image has the best focus. The total distance for detection 


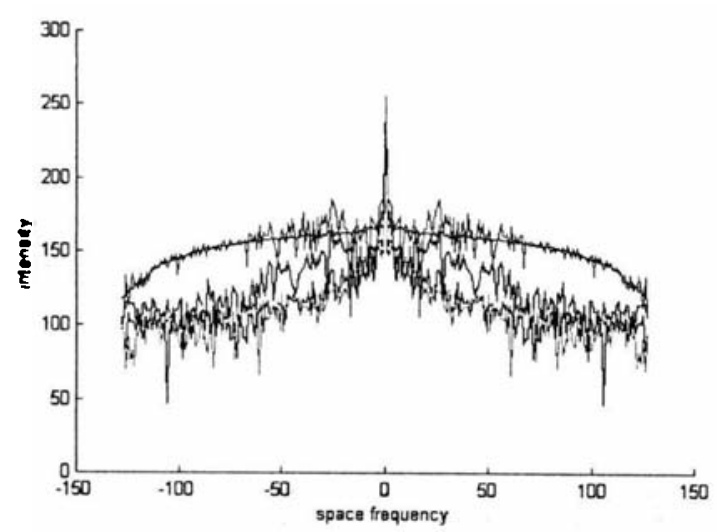

Fig.5 The frequency response of the image of Fig. 3

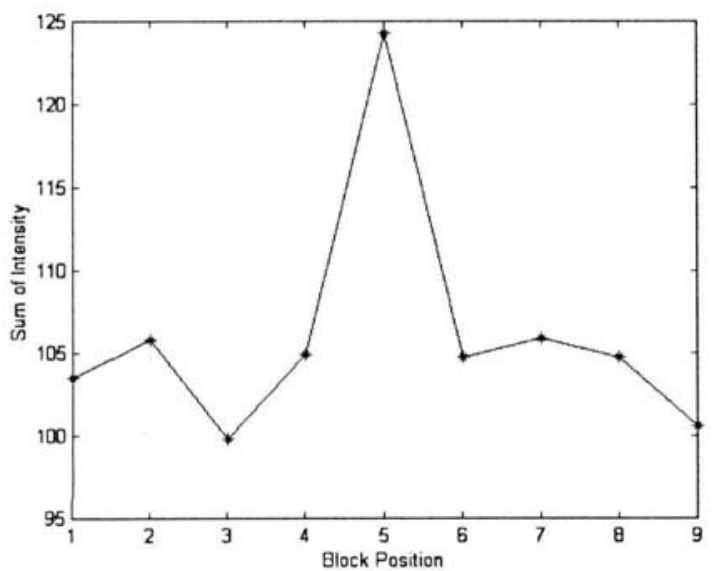

Fig.6 The relation of an image block and the sum of intensity

is 0.5 to 10 meter. In other word, 48 pictures would be grabbed within the testing area. The mapping distance of the corrected focus image is the measured result. For the detection of multi-point hindrance, the testing image should be divided to nine $(3 \times 3)$ medium-blocks. So, an image frame would have 9-distance message generated. The nine blocks of each image would be analyzed simultaneously and image-by-image to find the measured distance of each block, which are the testing distances of hindrance.

The image processing procedure is as follow:

a) Using equation (5) calculated $H_{0}$ by taking a clear photo $t_{0}$ for a specific object or view $p_{0}$ at a certain distance and letting $p_{0}=t_{0}$.

b) Adjusting the focus ring one scale, the image $t_{1}$ should be a little bit unclear. Let $p_{1}=t_{0}$ to calculate $H_{1}$ by equation (5).

c) Adjusting the focus ring one more scale, the image is unclear called $t_{2}$. Let $p_{2}=t_{0}$ to calculate $H_{2}$ by equation (S).

d) Repeat procedure iii for 48 times, 48 different

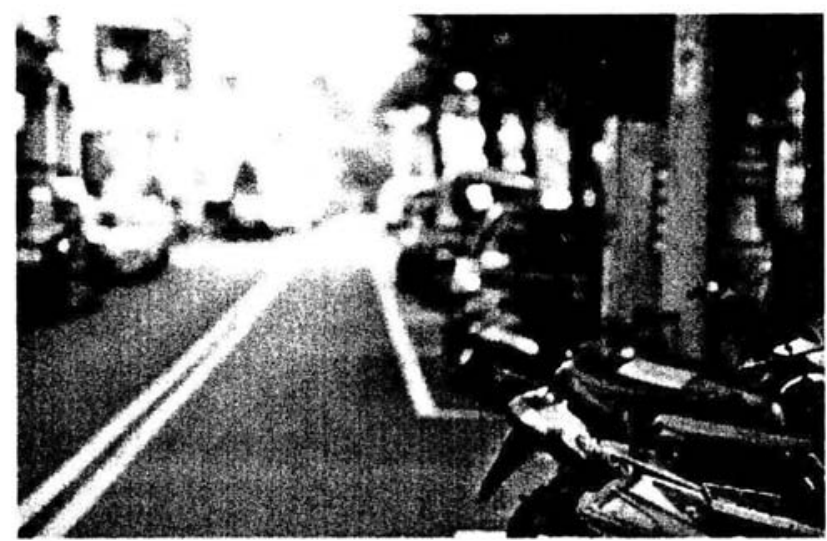

Fig.7 A real testing image

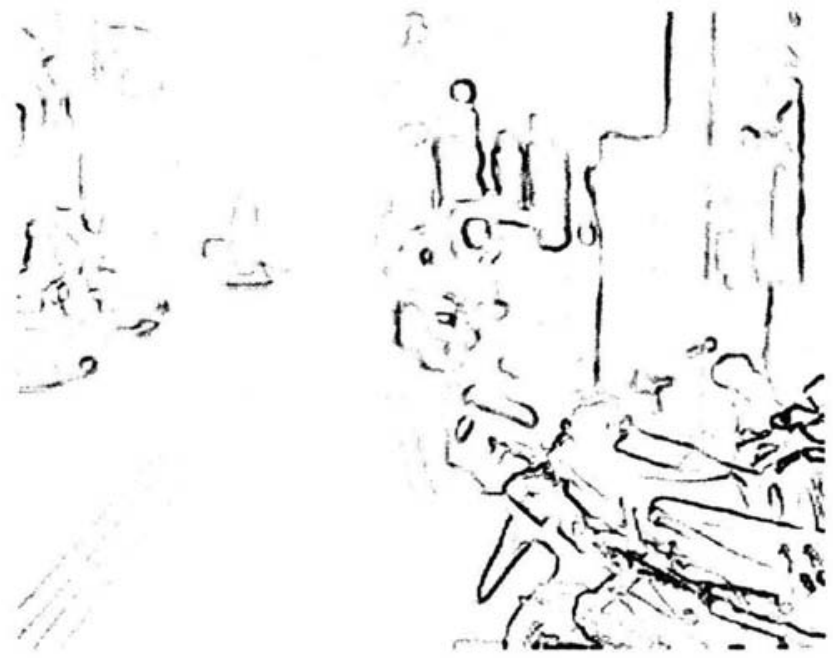

Fig.8 The result of edge enhancement

transform functions will be got, named $H_{1}, H_{2}$, $H_{3} \ldots \ldots H_{48}$.

e) The 48 same label blocks should be compared to find the best high frequency response one that is the right focus image block.

Fig.7 is an outdoor image processed by our propose method for proving the performance of the system. The image is converted into 256 gray scales and finds the skeleton as the edges feature by edge processing algorithm as shown in Fig.8. Divide the image into 9 medium blocks, from top to bottom, left to right, the order is named from i to 9 shown as Fig.9. Each block of the 9 medium blocks has been divided into 9 small blocks and label the small blocks from a to $i$. All the small blocks should be processed by Fourier transform to calculate the highest frequency response. The medium blocks of $7,8,9$ have most small blocks of high frequency response. Hence, the highest average intensity of medium blocks are the number $7,8,9$ blocks. The average intensities of all other medium blocks are lower than the number $7,8,9$ blocks, which are the right focus image blocks with the correct mapping dis- 


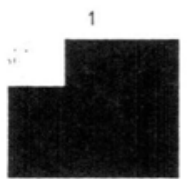

4

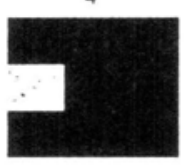

7

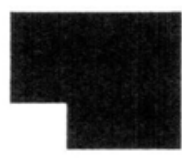

Fig.9 The sub-block of the testing image

tances as shown in Fig.10. In other word, our proposed method is to compare the spatial frequency of each specific medium block within 48 images to find the best high frequency response block and the mapping distance is the result. Because, a clear image has a better high frequency response than an out of focus image does. The distance data will be transmitted to the user's ear by a voice conversion device.

\section{CONCLUSION}

This paper presents a machine vision blind guide system. The system grabbed the image of front view and converted nine distance data to the blind by voice signal. A portable and useful blind guide system needs a very tiny computer and a long last battery. Hence, how to let the system small and power saving is one limitation of the system. This electronic blind guide system is worth to developed because the guide dogs are very expensive and not easy to maintain. A cane is cheaper but not possible to detect the area, which cannot reach. Recently, a 3D monitor is for blinds, which is read the screen by fingers has been developed. In the future, our system combines this kind monitor, when a blind use this guide system with a cane; the motion and orientation will be easier.

\section{ACKNOWLEDGMENT}

The author gratefully acknowledges financial support from the National Science Council of Taiwan, under Grant No. NSC-90-2213-E-167-010.

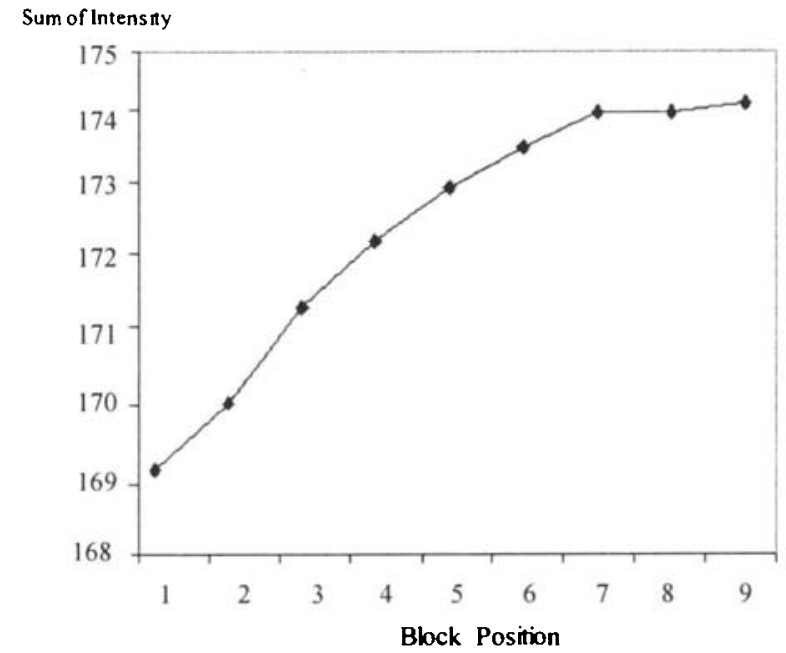

Fig.10 The testing result

\section{REFERENCES}

1. Gilman, G.D. "A Case in Point: Optometric or Psychology Problem?”,J. Am. Optom. Assoc., 52(7), Aug. 1981.

2. Swanson, W.J."Visually Related Leraning Disorders" J. Calif. Optom. Assoc. 1971; 34:343.

3. Kaisto, J. Kostamovarra, M. Manninen, and R. Myllya,"Optical range finder for $1.5-10 \mathrm{~m}$ distance,", Appl. 1983; 22:3258.

4. P. Chavel, and T.C. Strand, "Range measurement using Tallbot diffraction imaging of grating", Appl. Opt 23, 1984; 23: 862.

5. E. Hecht, A. Zajac, "Optics", Addison, Wesley, 1974.

6. Warren J. Smith, "Modern Optical Engineering", McGraw-Hill Interantional Editions, 1991.

7. Joseph W. Goodman, "Introduction to Fourier Optics", McGraw-Hill International Editions, Second edition, 1996.

8. J. M. Zurada, "Introduction to Artificial Neural Systems", West Info Access, 1992.

9. M. C. Fairhurst, "Computer Vision for Robotic System An Introduction “, Prentice Hall, 1988. 